\title{
Anomalies des autosomes et infertilité masculine
}

\author{
Yves RUMPLER, Odette GABREL-ROBEZ \\ Institut d'Embryologie, Université Louis Pasteur, Faculté de médecine, \\ 11 rue Humann, F-67085 Strasbourg Cedex, France.
}

\section{RESUME}

Il existe fréquemment une réduction de la gamètogenèse chez les sujets masculins porteurs de remaniements du nombre et de la structure des autosomes. En vue d'une étude de la méiose de ces patients, des biopsies testiculaires ont été effectuées chez des porteurs de translocations réciproques, de fusions centriques, d'inversions et d'extra chromosomes. Le rôle respectif du retard d'appariement des chromosomes remaniés et de leur association avec le bivalent sexuel dans la genèse des altérations de la spermatogenèse a été discuté.

Mots clés : Anomalie chromosomique - autosome - méiose - oligospermie - infertilité masculine.

\section{INTRODUCTION}

Les cytogénéticiens avaient montré depuis plusieurs décennies que les anomalies des chromosomes sexuels étaient souvent à l'origine de stérilités humaines. C'est ainsi que $1,5 \%$ de sujets présentant des oligospermies ou des azoospermies sont porteurs d'une anomalie du nombre ou de la forme des chromosomes sexuels. Outre l'altération de la fertilité, les différentes anomalies des chromosomes sexuels provoquent des malformations morphologiques caractéristiques qui ont permis de décrire toute une série de syndromes : syndrome de Turner, syndrome de Klinefelter, etc.
Les anomalies de structure équilibrées des autosomes réduisent aussi dans un grand nombre de cas la fécondité des individus qui en sont porteurs mais n'altèrent pas leur aspect morphologique. Elles agissent soit en provoquant des fausses couches à répétition, soit en perturbant la gamètogenèse, et plus spécialement la spermatogenèse. C'est ainsi que les fusions centriques et les translocations réciproques sont recensées 10 fois plus souvent chez les hommes hypofertiles que dans la population générale [5, 7]. Les effets délétères sur la gamètogenèse étant beaucoup plus importants chez l'homme que chez la femme, il est apparu qu'il devait exister une relation entre les autosomes remaniés et les chromosomes XY. Cet aspect a suscité un grand intérêt des biologistes en général et des andrologues en particulier. Des études morphologiques en microscopie électronique et des études expérimentales ont permis d'analyser d'une façon plus précise les effets sur la spermatogenèse de l'association des autosomes remaniés avec le bivalent sexuel.

\section{ETUDES DES COMPLEXES SYNAPTONEMAUX}

\section{ETUDES MORPHOLOGIQUES EN MICROSCOPIE ELECTRONIQUE DE CAS HUMAINS}

La technique de microspreading, mise au point par Moses [29], permet d'obtenir des spermatocytes I très étalés sur lesquels on peut colorer avec du nitrate d'Ag ou de 
l'acide phosphotungstique des structures protéiques qui interviennent dans l'appariement des chromosomes : les complexes synaptonémaux. Ces structures bien observables au microscope électronique apparaissent dans les spermatocytes I au stade zygotène et disparaissent après le pachytène. Elles visualisent les phases de l'appariement des chromosomes. Dans les 2 sexes les autosomes effectuent leur synapsis au zygotène pour former des bivalents et les crossing-over qui ont lieu durant le pachytène apparaissent sous forme de nodules de recombinaisons. Chez la femelle les 2 chromosomes X s'apparient sur toute leur longueur de la même façon que les autosomes, dès le stade zygotène. Chez le mâle, l'appariement des chromosomes $\mathrm{X}$ et $\mathrm{Y}$ ne débute qu'au stade pachytène et la synapsis ne se fait que sur la partie homologue de l'X et de l'Y. Il passe par 5 phases caractéristiques [37] permettant d'établir une chronologie: pachytène précoce, pachytène moyen, pachytène tardif (Figure 1).

Chez les porteurs de remaniements chromosomiques, il existe dans la plupart des cas un retard important de l'appariement des chromosomes remaniés. L'appariement n'est pas achevé au début pachytène et des zones non synapsées peuvent même persister jusqu'à la fin de ce stade.
1. Dans les translocations Robertsoniennes ou fusions centriques, les 2 chromosomes acrocentriques s'apparient avec le métacentrique issu du remaniement, pour former un trivalent.La synapsis débute aux extrémités distales des 3 chromosomes et progresse en direction des centromères. Les courts bras hétérochromatiques des 2 chromosomes acrocentriques restent longtemps non synapsés et ne s'apparient souvent qu'en fin de pachytène (Figure 2). Ces bras libres peuvent alors entrer en contact avec l'extrémité des chromosomes sexuels. Dans les cas où des chromosomes remaniés sont associés au chromosome X, l'aspect morphologique des autosomes au contact direct du bivalent sexuel est souvent modifié et ils prennent un aspect plus dense et plus irrégulier, semblable à celui que présentent l'X et l'Y. De ce fait, il est souvent impossible de distinguer ce qui est autosomique de ce qui est bivalent sexuel.

2. Dans les translocations réciproques, l'appariement des chromosomes forme un quadrivalent. Si aucun chromosome acrocentrique n'est impliqué dans la translocation, l'appariement débute par les 4 extrémités distales du quadrivalent et progresse en direction des centromères jusqu'à ce que la synapsis soit complète (Figure 2). Des
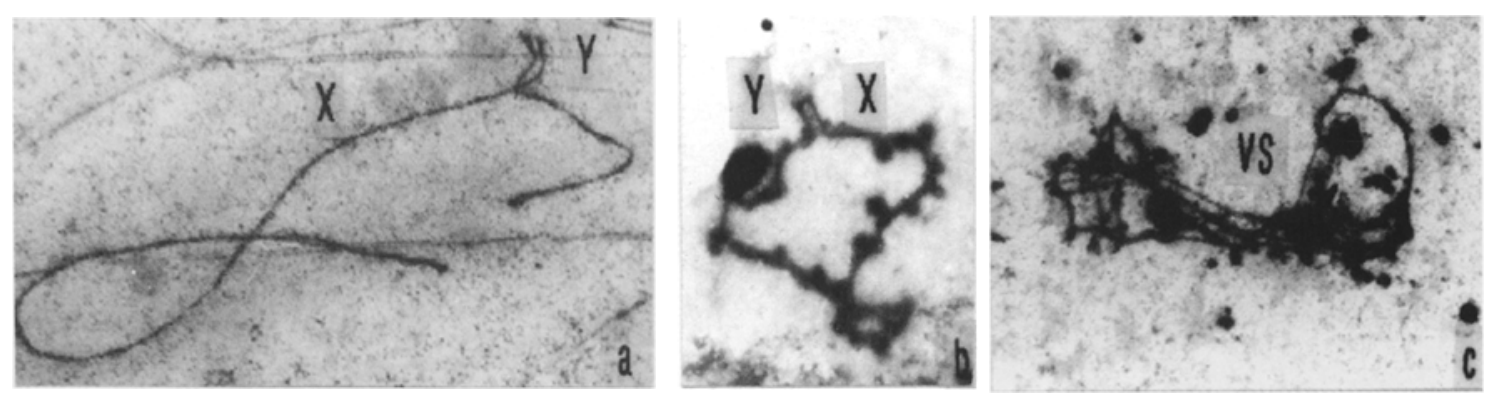

Figure 1 : Micrographies montrant 3 étapes de l'appariement de l'X et de l'Y durant le stade pachytène grâce aux complexes synaptonémaux (CS). a. Au début du stade pachytène le bivalent sexuel est uniquement synapsé sur une partie de sa longueur, le reste montre un aspect condensé. b. Au stade pachytène moyen les parties non synapsées sont souvent dédoublées en nombreux éléments présentant des excrescences. c. Au stade pachytène tardif, les axes du bivalent sexuel constituent un réseau complexe où on ne peut plus distinguer ce qui revient à l'X et à l'Y (vésicule sexuelle). VS = vésicule sexuelle. 
CHROMOSOMES

MITOTIQUES

1

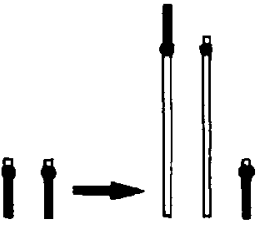

FUSION CENTRIQUE

CHROMOSOMES

MEIOTIQUES

DISPOSITION

THEORIQUE

DISPOSITIONS OBSERVEES

début

pachytène

fin

pachytène
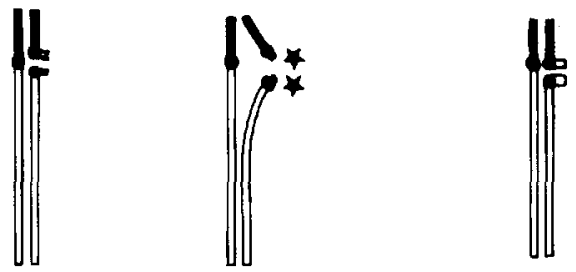

TRIVALENT

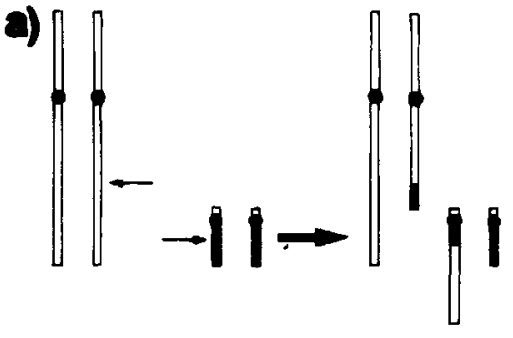

b)

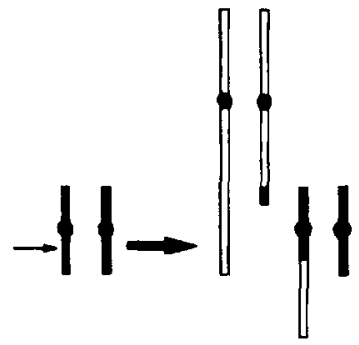

TRANSLOCATIONS RECIPROQUES
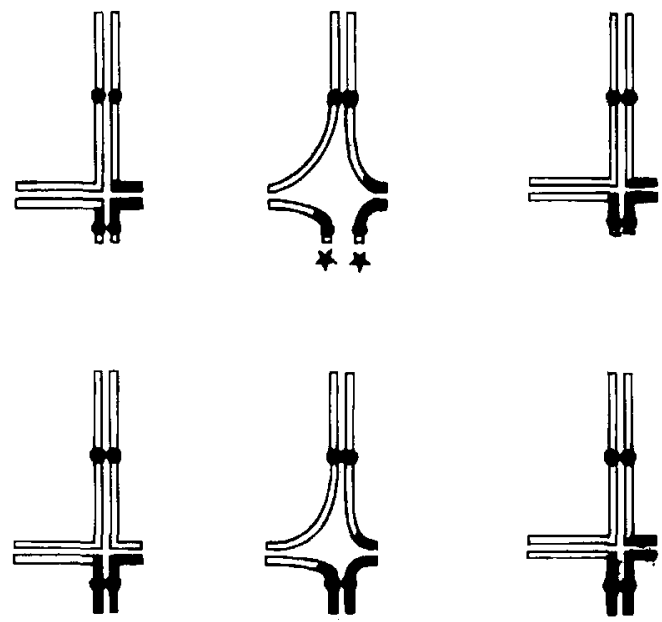

Figure 2 : Schéma indiquant la configuration méiotique dans les translocations Robertsoniennes (fusions centriques) et les translocations réciproques. Dans les translocations Robertsoniennes, les petits bras hétérochromatiques restent plus longtemps non appariés (astérisques) et peuvent entrer en contact avec le bivalent sexuel. Dans les translocations réciproques, lorsqu'un chromosome acrocentrique est impliqué dans la translocation, $a$. les petits bras hétérochromatiques restent également plus longtemps non synapsés (astérisques) et peuvent s'associer avec le bivalent sexuel. Dans les cas n'incluant pas de chromosomes acrocentriques, b. les 4 extrémités du quadrivalent sont synapsées et les associations avec le bivalent sexuel sont beaucoup moins fréquentes. 
zones non synapsées peuvent persister longtemps et la synapsis ne se termine parfois que tardivement (fin pachytène). Si au moins un chromosome acrocentrique est impliqué dans la translocation réciproque, la synapsis du quadrivalent ne débute qu'au niveau des bras distaux et les 2 extrémités proximales, hétérochromatiques, de la paire de chromosomes acrocentriques restent non synapsées durant une partie du pachytène (Figure 2). Ces bras du quadrivalent restés libres peuvent, comme pour les trivalents, entrer en contact avec le bivalent sexuel (Figure 3) et les autosomes peuvent présenter les mêmes modifications morphologiques que celles décrites pour les trivalents.

Il existe une relation quasi constante entre le pourcentage de spermatocytes où les chromosomes remaniés présentent des bras non appariés et la fréquence d'association de ces chromosomes avec le bivalent sexuel. Il existe également une relation fréquente entre la gravité de l'oligospermie [34, 24] et le pourcentage de spermatocytes où l'on observe une association entre les chromosomes remaniés et le bivalent sexuel (Tableau 1).

\section{ETUDE DE L'ACTIVITE TRANSCRIP. TIONNELLE DES CHROMOSOMES}

L'étude autoradiographique après incorporation d'uridine marquée dans les spermatocytes de ditiérentes espèces (criquets [14], souris [28, 38, 18], homme [36]) a montré que, durant le stade pachytène, l'activité transcriptionnelle des autosomes était importante alors que celle du bivalent sexuel était très réduite. Ceci avait amené LIFSCHYTZ et LINDSLEY [25] à proposer que, dans les cas de translocation X/autosome, une interaction entre l'autosome actif et le $\mathrm{X}$ inactif produirait des troubles métaboliques entraînant la mort des cellules.

Les mêmes phénomènes se produisent également dans les remaniements autoso-

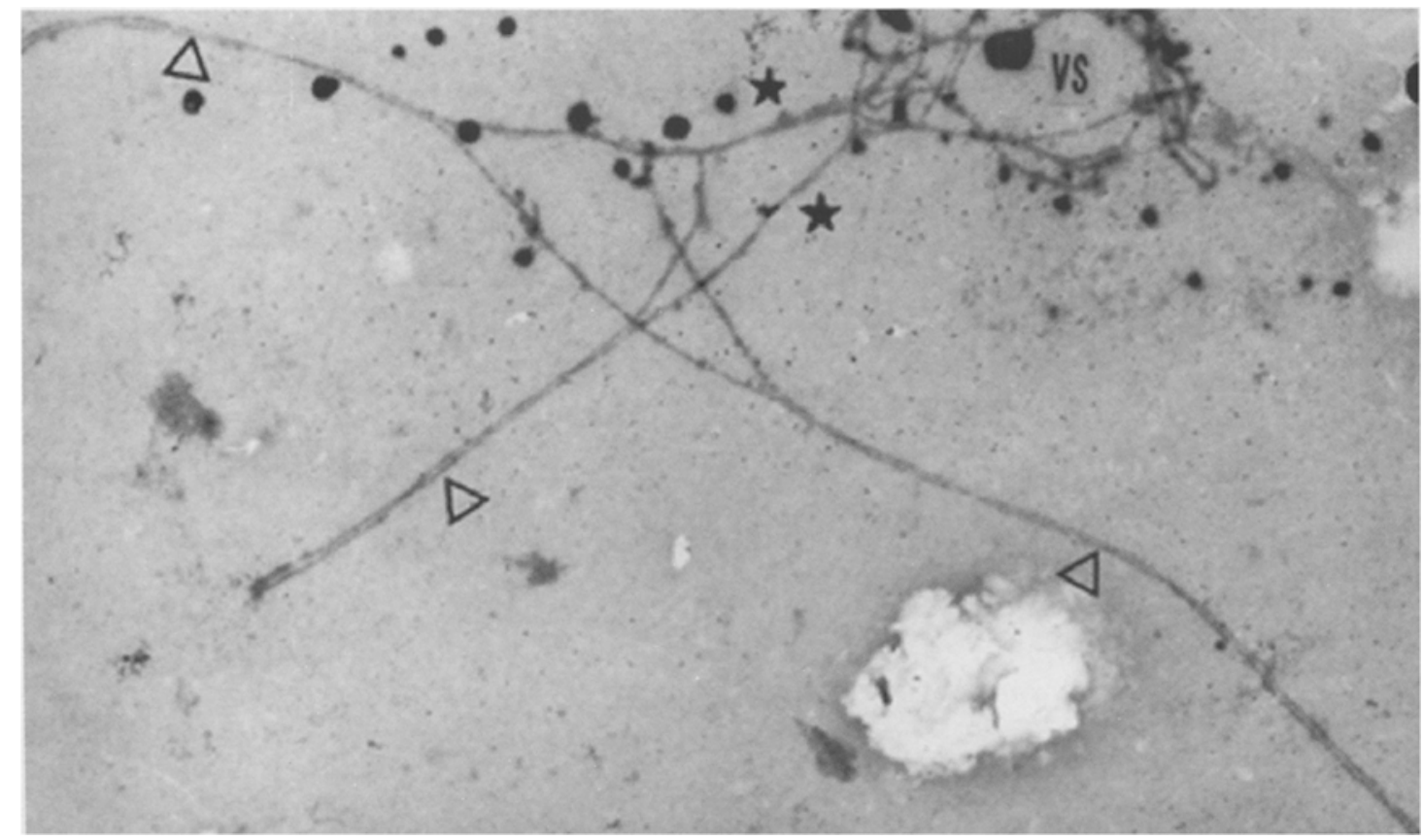

Figure 3 : Quadrivalent résultat d'une translocation réciproque : 3 bras du quadrivalent sont synapsés (tête de flèche), le 4e, comprenant les extrémités hétérochromatiques du chromosome acrocentrique, est resté non synapsé (astérisques) et est en contact avec le bivalent sexuel (VS). 


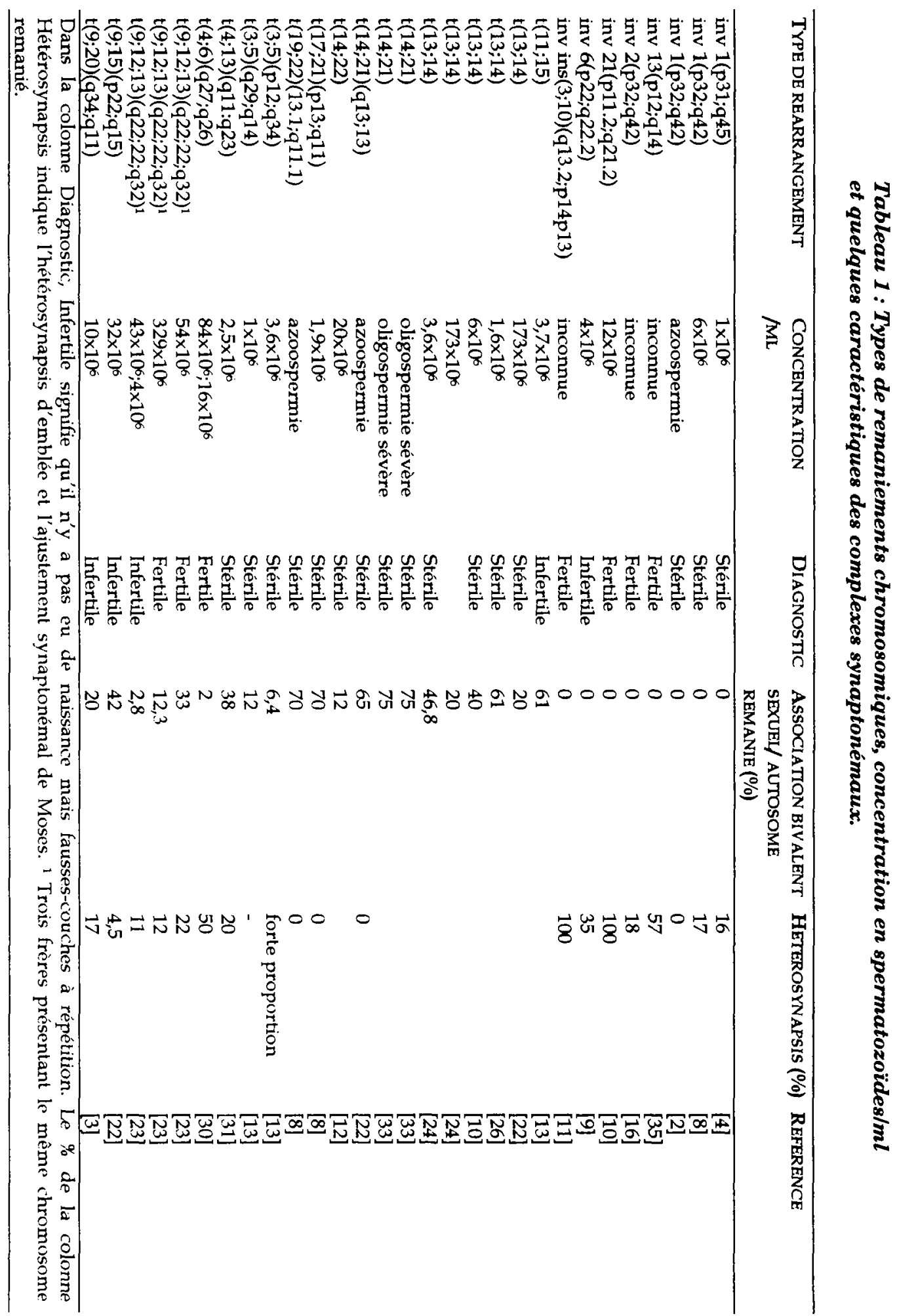


miques, lorsque les chromosomes remaniés s'associent au bivalent sexuel [6]. HoTTA et Chandley [15] ont montré que l'activité enzymatique due à des gènes liés au chromosome X était augmentée dans les spermatocytes de souris porteuses de translocation $\mathrm{X}$-autosome par rapport aux souris témoins. Ceci démontrerait la réactivation d'une partie des gènes normalement inactifs du $\mathrm{X}$ au contact de l'autosome transloqué.

JAAFAR et coll. [19] ont par contre montré par des études autoradiographiques, tant chez des souris porteuses de translocations $\mathrm{X}$-autosome que chez des souris porteuses de translocations réciproques où le quadrivalent est souvent associé au bivalent sexuel que l'activité transcriptionnelle des chromosomes $\mathrm{X}$ n'êtait pas significativement modifiée par rapport aux témoins, mais que celle des autosomes au contact du $\mathrm{X}$ était réduite de près de $50 \%$. La réduction était plus importante pour les segments directement au contact du X que pour les segments plus éloignés. Ceci démontrerait une extension de l'inactivation du X sur les autosomes transloqués sur le $\mathrm{X}$ ou associés à lui. L'étude morphologique des complexes synaptonémaux apporte également un argument indirect en faveur de cette hypothèse: si on admet un lien entre l'aspect particulier des complexes synaptonémaux du bivalent sexuel et l'état d'inactivation de ce dernier, par analogie les modifications morphologiques observées au niveau des autosomes remaniés associés au bivalent sexuel traduiraient une inactivation de ces éléments.

La possibilité d'une action se manifestant dans les 2 sens n'est pas exclue: inactivation de l'activité transcriptionnelle de gènes autosomiques et réactivation de certains gènes portés par le $\mathrm{X}$.

\section{COMPORTEMENT MEIOTIQUE DES EXTRA CHROMOSOMES}

Les chromosomes remaniés sont fréquemment associés au bivalent sexuel et lorsqu'ils restent libres ils sont souvent à proximité de ce dernier. Ceci traduit une "attraction" de ces chromosomes remaniés par le bivalent sexuel. Cette attraction s'exerce également à l'encontre d'autosomes supplémentaires qui forment des univalents durant le stade pachytène : le 21 supplémentaire des trisomies 21 est situé dans la vésicule sexuelle [21], les "extra chromosomes" de nature essentiellement hétérochromatique, qui ségrègent dans certaines familles où ils sont transmis par la mère, sont également situés à proximité immédiate du bivalent XY [20]. Il en est de même pour les chromosomes de nature entièrement hétérochromatique que l'on trouve normalement chez certaines espèces: les chromosomes $\mathrm{B}$, dont le nombre varie d'un individu à l'autre et que l'on trouve chez le renard ou certains rongeurs [171, ou la paire de chromosomes hétérochromatiques qui caractérise certains Gerbillidae [32]. Les causes de l'attraction exercée par le bivalent XY sur les éléments hétérochromatiques, qu'ils soient des chromosomes entiers comme les chromosomes B, ou les extrémités hétérochromatiques restées libres des acrocentriques remaniés (trivalents, quadrivalents) ou surnuméraires (trisomies) ne sont pas connues. Les conséquences de cette association sont variables selon le type de chromosome considéré.

Les chromosomes entièrement hétérochromatiques, comme les chromosomes B que l'on trouve à l'état normal dans certaines espèces, ne présentent aucune activité transcriptionnelle durant le stade pachytène et n'interferent pas avec l'inactivation normale du bivalent sexuel [171. Par contre, les chromosomes euchromatiques supplémentaires qui sont situés dans la vésicule sexuelle interfèrent avec elle, comme cela a été montré chez des souris trisomiques 113 où le fragment chromosomique euchromatique supplémentaire situé dans la vésicule sexuelle est partiellement inactivé [19]. On peut penser que l'altération profonde de la spermatogenèse chez les trisomiques 21 est due à des interactions de ce type qui perturbent le métabolisme des 
spermatocytes et provoquent la dégénérescence des cellules germinales. Les troubles de la gamètogenèse chez les porteurs d"extra chromosome" seraient plus légers car ces chromosomes semblent surtout constitués d'hétérochromatine et l'interaction bivalent sexuel/autosome serait bien plus faible, se rapprochant de ce que l'on observe dans le chromosome B.

\section{ROLE DES INVERSIONS}

Si l'hypothèse d'une interaction chromosome remanié/bivalent sexuel permet d'expliquer les oligospermies observées dans les translocations réciproques et Robertsoniennes, elle n'explique aucunement pourquoi les inversions s'accompagnent également d'une oligospermie chez un certain nombre de porteurs.
L'étude de toute une série d'inversions, tant chez la souris que chez l'homme, a montré que l'appariement du chromosome inversé durant le stade pachytène pouvait s'effectuer selon deux modalités.

Dans la plupart des cas, le segment inversé présente un retard d'appariement, suivi par la formation d'une boucle d'inversion qui disparaît en fin de pachytène par un alignement non homologue du segment inversé (ajustement synaptique de Moses (Figure 4a). Dans d'autres cas, en particulier lorsque le segment inversé est réduit [10], l'alignement non homologue du segment inversé se fait d'emblée et aucune boucle d'inversion n'est constituée (appariement hétérosynaptique) (Figure 4b). Quelles que soient les causes intervenant dans la survenue de l'une ou l'autre modalité, le segment
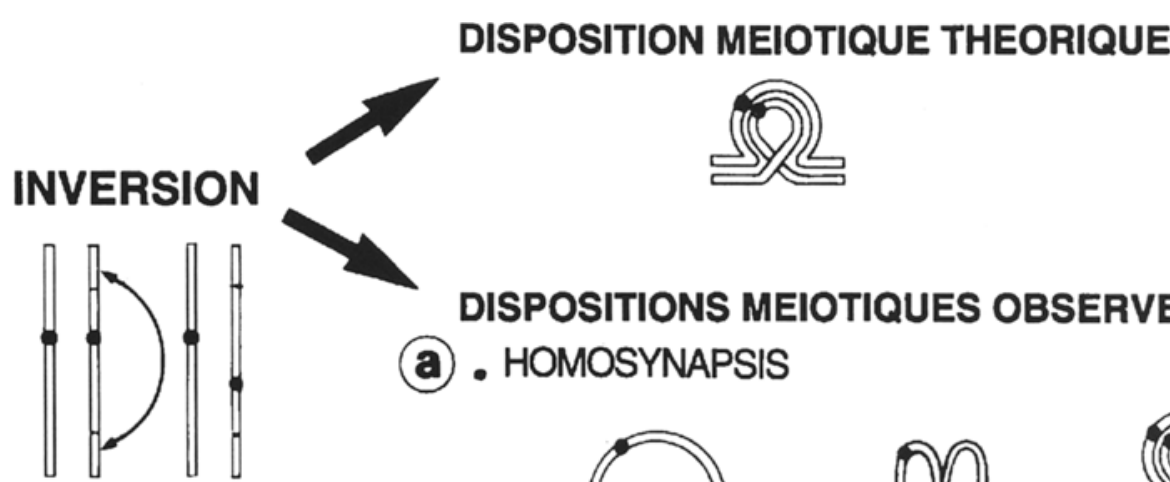

4

DISPOSITIONS MEIOTIQUES OBSERVEES

(a) . HOMOSYNAPSIS

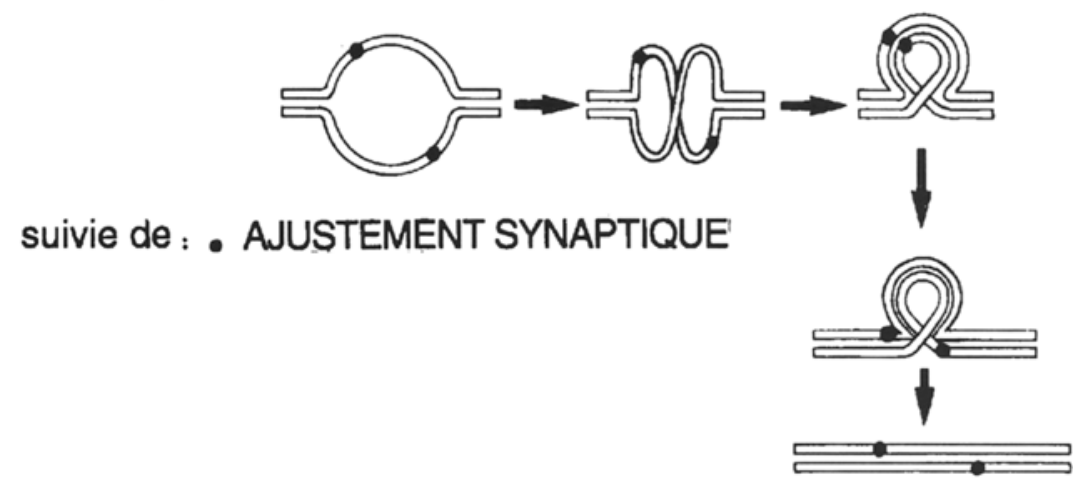

(b) - HETEROSYNAPSIS D'EMBLEE

Figure 4: Schéma indiquant les configurations méiotiques observées chez les porteurs d'inversion. a. Lorsqu'une boucle se forme, elle disparait en fin de pachytène par un ajustement synaptique. b. Dans certains cas, l'hétérosynapsis se produit d'emblée dès le début du stade pachytène et aboutit à un alignement des 2 chromosomes. 
inversé n'entre pas en contact avec le bivalent sexuel de sorte qu'on ne peut incriminer une interaction chromosome remaniél bivalent sexuel pour expliquer l'oligospermie observée. Selon certains auteurs [27] il existerait des sites d'appariement privilégiés répartis le long des chromosomes, et en cas de retard, ces sites non saturés, exerceraient une action délétère sur le métabolisme de la cellule. La relation existante entre la gravité de l'oligospermie observée et le pourcentage de spermatocytes présentant un retard de synapsis jusqu'à la fin du stade pachytène apporte un argument en faveur de cette hypothèse [1].

En conclusion, les remaniements chromosomiques des autosomes exercent une action délétère directe sur la gamètogenèse. Durant le stade pachytène les chromosomes remaniés présentent le plus souvent un retard d'appariement et les troubles de la gamètogenèse sont d'autant plus importants que la proportion de spermatocytes présentant un tel retard est élevé. Dans le cas des translocations réciproques ou Robertsoniennes, ou des trisomies, les chromosomes anormaux ont tendance à entrer en contact avec le bivalent sexuel et interagissent avec lui. L'interaction entre un bivalent sexuel dont l'activité transcriptionnelle est réduite et un autosome dont l'activité transcriptionnelle est importante se traduit par une extension de l'inactivation de l'X à l'autosome entré en contact avec lui et peut être aussi par une réactivation de certains gènes du chromosome $X$. Cette interaction serait en partie responsable de la dégénérescence des cellules germinales après le stade pachytène.

Dans un certain nombre de cas où n'existe pas cette relation entre le pourcentage d'association et la gravité de l'atteinte de la spermatogenèse, les troubles de synapsis des chromosomes transloqués sont à prendre en considération, tout comme pour les inversions, bien qu'on ne connaisse pas, à l'heure actuelle, par quel mécanisme précis ce facteur agit. Des études ultérieures faisant appel à ces techniques d'hybridation in situ par des sondes d'ARN permettront peut-être d'aller plus avant.

Remarque : Il apparaît ainsi qu'un certain nombre de cas d'oligospermie importante sont d'origine chromosomique et ne peuvent actuellement être améliorés par un traitement médical. L'examen méiotique a donc un intérêt pronostique certain puisque la fréquence des spermatocytes présentant des asynapsis des associations entre autosome et chromosomes sexuels permet de déduire l'irréversibilité de la situation. L'intérêt de l'étude méiotique dans les cas d'oligospermie est encore accru du fait qu'un certain nombre de patients, non porteurs d'anomalies chromosomiques, présentent également des troubles importants de l'appariement d'un grand nombre de chromosomes et que les mêmes conclusions pronostiques peuvent en être tirées.

Remerciements : Les auteurs remercient Mme M. Lavaux pour le travail dactylographique et le Dr B. Brun pour le travail photographique. Ce travail a été réalisé grâce au soutien financier de la Fondation Médicale pour la Recherche Française.

\section{REFERENCES}

1. AN EUROPEAN COLLABORATIVE STUDY. : The meiotic pairing behaviour in human spermatocytes carrier of chromosome anomalies and their repercussions on reproductive fitness. I. Inversions and Insertion. Prepared by $\mathrm{O}$ GabrielRobez and $\mathrm{Y}$. Rumpler. Ann. Génét, in press.

2. BATANIAN J., HULTEN M.A. : Electron microscopic investigations of synaptonemal complexes in an infertile male carrier of a pericentric inversion inv (1) (p32; q42). Regular loop formation but defective synapsis including a possible interchromosomal effect. Him. Genet., 1987, $76: 81$ 86.

3. CHANDLEY A.C., SPEED R.M., MCBEATH S., HARGREAVE T.B. : A human $9 j 20$ reciprocal translocation associated with male infertility analyzed at prophase and metaphase I of meiosis. Cytogenet. Cell Genet., 1986, $41: 145-153$.

4. CHANDLEY A.C., MCBEATH S., SPEED R.M., YORSTON L., HARGREAVE T.B. : Pericentric inversion in human chromosome 1 and the risk for male sterility. J. Med. Genet., 1987, 24 : 325334.

5. DUTRILLAUX B., GUEGEN J. : Etude méiotique et mitotique dans un cas de translocation $t(5 ; \mathrm{Y})$. Hum. Genet., 1971, 27 : 241-245. 
6. FOREJT J., GREGOROVA S., GOETZ P. : XY pair associates with the synaptonemal complex of autosomal male sterility translocation in pachytene spermatocytes of the mouse (Mus musculus). Chromosoma, 1981, $82: 41-53$.

7. FRACCARO M. : Chromosome abnormalities and gamete production in man. Differentiation, 1983, 23 (Suppl.) : S40-S43.

8. GABRIELROBEZ O., RATOMPONIRINA C., RUMPLER Y., LE MAREC B., LUCIANI J.M., GUICHAOUA M.R : Synapsis and asynaptic adjustment in an human infertile male heterozygous for a pericentric inversion in chromosome 1 . Hum. Genet., 1986, 72 : 148-152.

9. GABRIELROBEZ O., RATOMPONIRINA C., CROQUETTE M., MAETZ J.L., COUTURIER J., RUMPLER Y. : Reproductive failure and pericentric inversion in man. Andrologia, 1987, 19 (6) : 662669.

10. GABRIELROBEZ O., RATOMPONIRINA C., CROQUETTE M., COUTURIER J., RUMPLER Y. : Electron microscopic investigations of synaptonemal complexes in an subfertile man carrier of a pericentric inversion in chromosome 21: heterosynapsis without previous homosynapsis. Cytogenet. Cell Genet. 1988, $48: 84-87$.

11. GOLDMANN A.S.H., MARTIN R.H., JOHANNISSON R., GOULD C.P., DAVISON E.V., EMSLIE J.E., BURN J., HULTEN A.M. Meiotic and sperm chromosome analysis in a male carrier of an inverted insertion ( $3 ; 10)$ (ql3.2; pl4; pl3). J. Med. Genet., 1992, 29, 460-464.

12. GUICHAOUA M.R., QUACK B., SPEED R.M., NOEL B., CHANDLEY A.C., LUCIANI J.M. : Infertility in human males with autosomal translocations. Hum. Genet., 1990, 86: 162-166.

13. GUICHAOUA M.R., SPEED R.M., LUCIANI J.M., DELAFONTAINE D., CHANDLEY A.C. : Infertility in human males with autosomal translocations. Cytogenet. Cell Genet., 1992, 60 : 96-101.

14. HENDERSON S.A. : RNA synthesis during male meiosis and spermiogenesis. Chromosoma, 1964, 15: 345-366.

15. HOTTA Y., CHANDLEY A.C. : Activities of X-linked enzymes in spermatocytes of mice rendered sterile by chromosomal aberrations. Gam. Res., $1982,6: 65-72$.

16. HULTEN M. SAADALLAH N. BATANIAN J. : Meiotic chromosome pairing in the human male: Experience from surface spread synaptonemal complexes. Chromosome Today, 1987, $9: 218-229$.

17. ISHAK B., JAAFAR H., RUMPLER Y. : Absence of transcriptional activity of the B-chromosomes of Apodemus peninsulae during pachytene stage. Chromosoma, 1991, $100: 278-281$.
18. JAAFAR H., GABRIEL-ROBEZ O., RUMPLER Y. Pattem of ribonucleic acid synthesis in vitro in primary spermatocytes from mouse testis carrying a $\mathrm{X}$-autosome translocation. Chromosoma, 1989, 98 : 330-334.

19. JAAFAR H., GABRIELROBEZ O., RUMPLER Y. : Chromosomal anomalies and disturbance of transcriptional activity at the pachytene stage of meiosis: Relationships to male sterility. Cytogenet. Cell Genet., 1993, 64 : 273-280.

20. JAAFAR H., GABRIELROBEZ O., VIGNON F., FLORI E., RUMPLER Y. : Supernumerary chromosomes and spermatogenesis in a human male carrier. Hum. Genet. 1994 (in press).

21. JOHANNISSONN R., GROPP A., WINKING H., COERDT W, REHDER H., SHWINGER E. : Down's syndrome in the male. Reproductive pathology and meiotic studies. Hum. Genet., 1983, 63 : 132-138.

22. JOHANNISSON R., LOHRS U., WOLTI H.H., SCHWINGER E. Two ditierent XY quadrivalent associations and impairment of fertility in men. Cytogenet. Cell Genet., 1987, 45: 222-230.

23. JOHANNISSON R., LOHRS U., PASSARGE E. : Pachytene analysis in male heterozygous for a familial translocation $(9 ; 12 ; 13)(\mathrm{q} 22 ; \mathrm{q} 22$; q3V ascertained through a child with partial trisomy 9. Cytogenet. Cell Genet., 1988, $47: 160$ 166.

24. JOHANNISSON R., SCHWINGER E., WOLTI H.H., VOM ENDE V., LOHRS U. : The etiect of 13j14 Robertsonian translocations on germ-cell differentiation in fertile males. Cytogenet; Cell Genet., 1993, $63: 161-155$.

25. LIFSCHYTZ E., LINDSLEY D.L. : The role of Xchromosome inactivation during spermatogenesis. Proc. Natl. Acad. Sci. USA, 1972, 69 : 182-186.

26. LUCIANI J.M., GUICHAOUA M.R., MATTEI M.A., MORAZZANI M.R. Pachytene analysis of a man with a 13q;14q translocation and fertility. Behaviour of the trivalent and non-random association with the sex vesicle. Cytogenet. Cell Genet., $1984,38: 14-22$.

27. MIKLOS G.L.G. : Sex chromosome pairing and male fertility. Cytogenet. Cell Genet., 1974, 13: 558-577.

28. MONESI V. : Synthetic activities during spermatogenesis in the mouse. Exp. Cell Res., 1965, 39 : 197-224.

29. MOSES M.J. : Microspreading and the synaptonemal complex in cytogenetic studies. In : Chapelle A. de la, Sorsa M. eds. Chromosome Today. Elsevier/North-Holland, Amsterdam, 1977, vol. $6: 71$ 82. 
30. PERDIGO DE A., GABRIEL-ROBEZ O., RATOMPONIRINA C., RUMPLER Y. : Synaptonemal complex analysis in a human male carrier of a $4 ; 6$ translocation: heterosynapsis without previous homosynapsis. Hum. Genet., 1991, 86 : 279-282.

31. PERDIGO DE A., GABRIELROBEZ O., RUMPLER Y. : Analysis of synaptonemal complexes in a heterozygous human male carrier of a reciprocal translocation involving an acrocentric chromosome: heterosynapsis without previous homosynapsis. Hum. Genet., 1991, 87 : 602-606.

32. RATOMPONIRINA C., VIEGAS-PÉQUIGNOT E., PETTER F., DUTRILLAUX B., RUMPLER Y. : Synaptonemal complex study in some species of Gerbillidae without heterochromatin interposition. Cytogenet. Cell Genet., 1989, $52: 23-27$.

33. ROSENMANN A., WAHRMAN J., RICHLER C., VOSS R., PERSITZ A., GOLDMAN B. : Meiotic association between the $X Y$ chromosomes and unpaired autosomal elements as a cause of human male sterility. Cytogenet. Cell Genet., 1985, 3g : 19-29.

34. RUMPLER Y., GABRIEL-ROBEZ O., RATOMPONIRINA C. Autosomal rearrangements and reproductive failure in man. In: Progress in reproductive biology and medicine, vol. 15, series editor $M$. L'Hermite, Brussels. Diagnosing Male Infertility. New possibilities and limits. Volume editors Giovanni M. Colpi Milan, Diego Pozza, Rome. Karger, Basel 1992 : 164177.

35. SAADALLAH N., HULTEN M. EM : Investigations of surface spread synaptonemal complexes in a human male carrier of a pericentric inversion inv(13) (pl2ql4): the role of heterosynapsis for spermatocyte survival. Ann. Hum. Genet., 1986, 50, 369-383.

36. SAUSSINE C., GABRIEL-ROBEZ O., RUMPLER Y. : Pattern of ribonucleic acid synthesis in human primary spermatocytes. Andrologia, in press.

37. SOLARI A.J. : Synaptonemal complexes and associated structures in microspread human spermatocytes. Chromosoma, 1980, 81 : 315-317.

38. SPEED R.M. : Abnormal RNA synthesis in sex vesicles of tertiary trisomic male mice. Chromosoma, 1986, 93: 267-270.

\section{ABSTRACT}

\section{Autosomal rearrangements and} male infertility

\section{Yves RuMPLER, Odette GABRIEL-Rodez}

Institut d'embryologie, 11 rue Humann, 67085 Strasbourg Cedex

Spermatogenesis impairment is frequently associated with autosomal rearrangements. A meiotic study was performed on testicular biopsies of men ascertained through primary fertility and found to be heterozygous for reciprocal translocation, Robertsonian translocations, inversions and extra chromosomes. Pairing failure at meiosis as well as intimate associations between rearranged autosomes and the $\mathrm{XY}$ bivalent have been discussed as a cause of gametogenic arrest.

Key words : Autosomal rearrangement - autosome - meiosis - oligospermia - male infertility. 